\title{
Ist die Feminisierung der Medizin eine Antwort auf den drohenden Ärztemangel?
}

Barbara Weil

Leiterin Abteilung Gesundheitsförderung und Prävention der FMH
Korrespondenz:

Barbara Weil

FMH

Elfenstrasse 18

CH-3000 Bern 15

barbara.weil@fmh.ch
Zwar war es für Frauen in der Antike und im Frühmittelalter nicht üblich, jedoch sehr wohl möglich, an Akademien zu studieren und zu lehren. Berichte über prominente Ärztinnen, Architektinnen und andere weibliche Gelehrte finden sich bereits im alten Ägypten, dem antiken Griechenland oder dem römischen Reich.

In der mittelalterlichen Feudalgesellschaft, die zunehmend kirchlichen Vorgaben folgte, gewährte man Frauen jahrhundertelang nur dann Zugang zu Bildung, wenn ihre Familie dem Kloster eine Mitgift übergab. Medizinische Studien und Lehre waren für Frauen eine der letzten Möglichkeiten, sich akademisch zu betätigen. Frühester Wegbereiter des Umschwungs zugunsten der Schulbildung von Mädchen und Frauen war Jan Amos Comenius, der Bischof der böhmisch-mährischen Brüdergemeinde: «Omnes, omnia, omnino» alle sollen alles allumfassend lernen dürfen!

Bis Frauen aber tatsächlich freien Zugang zu universitärer Bildung erhielten, musste noch ein steiniger Weg beschritten und unzählige Vorurteile ausgeräumt werden. Bevor sie zugelassen wurden, stritt man sich über Jahrhunderte darüber, ob Frauen von ihrer geistigen Leistungsfähigkeit und körperlichen Verfassung her überhaupt für ein Studium geeignet wären. Heute werden solche Aussagen kopfschüttelnd belächelt - längst sind Frauen in medizinischen Studiengängen in der Überzahl, es wird von einer Feminisierung der Medizin gesprochen [1]. Stimmrecht, Berufswahl, nach der Heirat nicht auf das Einverständnis des Ehemannes angewiesen zu sein, wenn frau arbeiten möchte, ein eigenes Bankkonto - all das ist heute für Frauen selbstverständlich und nicht mehr aus unserem Alltag wegzudenken.

Auch die Weiterbildungsordnung (WBO) gibt Frauen die Möglichkeit, ihre fachspezifische Weiterbil-

\section{Art. 32 Voll- und Teilzeitarbeit}

- Bis zu $50 \%$ der fachspezifischen Weiterbildung kann in Teilzeit absolviert werden, es sei denn, das Weiterbildungsprogramm lässt einen höheren Anteil zu.

- Nicht fachspezifische Weiterbildung kann vollumfänglich in Teilzeit absolviert werden.

- Der Umfang der Teilzeitbeschäftigung muss mindestens $50 \%$ eines Vollpensums entsprechen. In Teilzeit absolvierte Weiterbildung wird anteilmässig angerechnet.

- Für Facharzttitel, zu deren Erwerb keine fachspezifische Weiterbildung vorgeschrieben ist, wird der zulässige Anteil der Teilzeit im Weiterbildungsprogramm geregelt. dung in Teilzeit zu absolvieren (siehe Kasten). So gesehen, ist Medizin ein ideales Frauenfach und insbesondere im Hinblick auf den drohenden Ärztemangel scheint zumindest eine Lösung in Sicht. Vergleicht man die auf dem Papier gegebenen Möglichkeiten mit der heutigen Realität, wirft dies Fragen auf, die es zu beantworten gilt, will man sich dem in der Schweiz abzeichnenden Ärztemangel wirkungsvoll begegnen [2, 3, 4].

- Sind die Weiterbildungsmöglichkeiten und Anstellungsbedingungen den heutigen Bedürfnissen von Ärztinnen angepasst oder basieren sie noch auf den althergebrachten männlichen Arbeitsbiografien und Rollenbildern?

- Werden in allen Fachgebieten tatsächlich flexible Teilzeitstellen auf allen Hierarchiestufen angeboten (z.B. Innere Medizin, Chirurgie, Orthopädie usw.)?

- Spitalinterne Kindertagesstätten für das Pflegepersonal sind heute praktisch Standard - doch: Gibt es genügend (finanzierbare) Kindertagesstätten, deren Öffnungszeiten den Arbeitszeiten von Ärztinnen und Ärzten angepasst sind (Frühdienst Spätdienst)?

- Ändern sich Haltungen und Meinungen zu Mutterschaft/Familie und Karriere oder verbleiben sie im bisherigen «Kinderkriegen und Karriere sind inkompatibel»?

- Werden genügend angepasste Mentoring-Programme angeboten?

Die junge Ärztegeneration verfolgt ihre berufliche Karriere nicht mehr bedingungslos - auf Kosten der Familie. Auch Väter möchten sich mehr um die Familie kümmern - tatsächliche Gleichberechtigung ist möglicherweise eine der Antworten.

\section{Literatur}

1 Kraft E, Hersperger M. Ärzteschaft in der Schweiz - die Feminisierung der Medizin. Schweiz Ärztezeitung. 2009;90(47):1823-5.

2 Buddeberg-Fischer B, Stamm M. The medical profession and young physicians' lifestyles in flux: challenges for specialty training and health care delivery systems. Swiss Med Wkly. 2010;140:w13134.

3 Stamm M, Buddeberg-Fischer B. How do physicians and their partners coordinate their careers and private lives? Swiss Med Wkly. 2011:141w13179.

4 Buddeberg-Fischer B, Stamm M, Buddeberg C, Bauer G, Hämmig O, Knecht M. The impact of gender and parenthood on physicians' careers - professional and personal situation seven years after graduation BMC. Health Services Research. 2010;10(40). 